\title{
Role of oligo(malic acid) on the formation of unilamellar vesicles
}

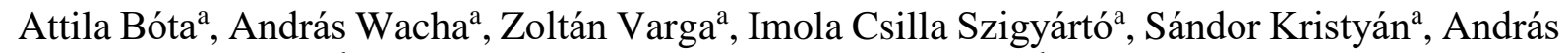
Lőrincz ${ }^{\mathrm{a}}$, Pál Szabób, Miklós Kálmán ${ }^{\mathrm{c}}$, Lívia Naszályi-Nagy ${ }^{\mathrm{a}, \mathrm{d}}$, Judith Mihály ${ }^{\mathrm{a}, *}$

anstitute of Materials and Environmental Chemistry, Research Centre for Natural Sciences, Hungarian Academy of Sciences, 1117 Budapest, Magyar tudósok körútja 2, Hungary

${ }^{\mathrm{b}}$ Institute of Organic Chemistry, Research Centre for Natural Sciences, Hungarian Academy of Sciences, 1117 Budapest, Magyar tudósok körútja 2, Hungary

${ }^{c}$ Nanochem Ltd., 6724 Szeged, Mars tér 7, Hungary

${ }^{\mathrm{d}}$ Department of Organic and Macromolecular Chemistry, Ghent University, 9000 Ghent, 281

Krijgslaan, Belgium

*Corresponding author:

mihaly.judith@ttk.mta.hu

\begin{abstract}
Stable unilamellar dipalmitoylphosphatidylcholine vesicles were produced by using oligo(malic acid) and cholesterol. Detailed physico-chemical characterization prove that by using oligo(malic acid) the substitution of PEGylated lipids for sterically stabilization comes possible. The polymer molecules cover the outer surface of spherical-shaped vesicles, and an asymmetrical composition occurs in the two leaflets of the phospholipid bilayer. The oligo(malic-acid) and cholesterol are enriched in the outer side assuring the stabilization of vesicles. Cholesterol plays an important role in the self-assembly of components as it makes the entering of oligomers possible deep into the polar head-region of lipids. The presence of oligo(malic acid) molecules does not induce degradation by hydrolysis of lipid molecules but the vesicle system turns into a sensitive form giving a possibility for $\mathrm{pH}$ sensitive targeting. Preliminary investigation on the investigated oligo(malic acid)-stabilized vesicles do not show any toxic effect promising their applicability in the field of liposomal drug delivery.
\end{abstract}

\section{Introduction}

The long-term circulation of sterically stabilized vesicles (SSV) in the vascular system is typically achieved by anchoring biocompatible polymer molecules onto their surfaces [1-3]. These small $(\leq 100 \mathrm{~nm})$, unilamellar vesicles are typical nanocarriers for therapeutic drugs and are already widely used in clinical practice [4]. Poly(ethylene-glycol), the commonly used biopolymer responsible for stabilization is covalently bonded to the head group of phospholipids. From these compounds the lipid part is embedded into the bilayer while the PEG chain covers the outer surface of the bilayer. These properties result in a biocompatible, "stealth" character of the whole object that hides them from the immune system. The first medical product based on SSVs was the liposomal doxorubicin, which was followed by many other formulations dominantly dedicated for cancer therapies [5]. Further modifications of SSVs provide broad possibilities in biological, pharmacological, cosmetic and medical applications [6, 7]. The usage of PEGylated SSVs is widespread, although it can cause a complement activation-related pseudo-allergy (CARPA) in some patients, resulting in dramatic cardiovascular and clinical abnormalities [8]. Consequently, in order to achieve fully 
biocompatible SSVs without any side-effect, great scientific effort is invested in research worldwide. It has been found that biopolymers used as nanoconjugate delivery systems can form a compact shell on the vesicles. For example, Rinaudo et al. have studied the stabilization of liposomes by polyelectrolytes like chitosan and hyaluronan [9, 10]. Without doubt, hyaluronan, as a fully biocompatible biopolymer and a constituent of the extracellular biomatrix is an effective molecule to assure the long circulation time of vesicles [11-13].

The multifunctional, nontoxic and nonimmunogenic poly(L-glutamic acid), poly(L-aspartic acid) and poly(L-malic acid) (PMLA) are also prominent biopolymer constituents of nanoconjugates [14]. PMLA is a fully biocompatible polyester that is degradable by hydrolysis, leading to the production of the nontoxic malic acid, which also participates in the citrate-circle. A further advantage of PMLA is that it contains carboxylic groups where various bioactive ligands can be attached. Numerous synthesis routes are known to produce both racemic and optically active PMLAs [15-17]. In order to reveal the nature of the interactions between PMLA and the cell membrane surfaces, the changes in thermotropic and structural behaviour of fully hydrated multilamellar dipalmitoylphosphatidylcholine (DPPC) system was studied in the presence of low molecular weight PMLA [18]. It was found that PMLA attaches to the headgroups of the phospholipids through hydrogen bonds between the free hydroxyl groups of PMLA and the phosphodiester groups of DPPC. The acidity of PMLA is not dominant and it does not causes hydrolysis in the lipid system $[19,20]$. Low molecular weight PMLA, however, induces a change on the electrostatic state of lipid headgroup region, even in the presence of buffer in the solvent, whereby uncorrelation between the lamellae can occur indicating the possible utilization of this charged polymer in stabilization of vesicles. Consequently we have constructed a new type of unilamellar vesicles with oligo(malic acid) for the purposes of drug delivery. Here we show the complex characterization - applying morphological, structural and theoretical calculation methods - of these vesicles and elucidate the role of each constituent in the colloidal stabilization of the system.

\section{Materials and methods}

Synthetic 1,2-dipalmitoyl-sn-glycero-3-phosphocholine (DPPC, > 99\% purity) was purchased from the NOF Corporation (Japan). Phosphate buffered saline (PBS) and cholesterol (> 99\% purity) were obtained from Sigma-Aldrich. These materials were used without further purification. The low-molecular weight oligo(malic acid) (abbreviated further on as OMLA) was purchased from Nanochem Ltd. (Szeged, Hungary) synthetized by the method of Kajiyama et. al. [21,22].

\section{Preparation of vesicle systems}

Concentrated hydrated samples (20 wt\% lipid), forming multilamellar vesicles, were made to elaborate the proper preparation protocol. The weight ratio of OMLA to lipid was 0.1 . The samples were prepared using two different routes: i) the main components - lipid and OMLA - were mixed in solid powder state and hydrated in $10 \mathrm{mM}$ PBS buffer $(7.4 \mathrm{pH})$; ii) the dry lipid was hydrated with a solution of OMLA prepared with $10 \mathrm{mM}$ PBS buffer. The resulting viscous suspensions were homogenized by cooling and heating cycles $\left(4^{\circ}-50^{\circ} \mathrm{C}\right)$ applied twenty times.

The unilamellar sterically stabilized vesicles (SSVs) containing also cholesterol were prepared by lipid film hydration and extrusion method [23] in small $(2 \mathrm{w} / \mathrm{w} \%)$ lipid concentration. Solid DPPC and cholesterol (3:1 molar ratio) were dissolved in a chloroform:methanol $(2: 1 \mathrm{v} / \mathrm{v}$ ratio). The solvent was then evaporated at $40^{\circ} \mathrm{C}$ and the dry film was kept in vacuum overnight to remove traces of the solvent. The lipid films were hydrated with OMLA solutions $(10 \mathrm{mM}$ 
PBS made of ultrapure water $(18 \mathrm{M} \Omega \cdot \mathrm{cm})$ corresponding to 0.05 and 0.1 OMLA/DPPC weight ratios. Weight ratio was used to characterize the composition because of the uncertainty of OMLA molecular weight. Taking into consideration the LC-MS analysis, the 0.05 and $0.1 \mathrm{w} / \mathrm{w}$ ratios correspond to approx. 0.05 and $0.1 \mathrm{~mol} / \mathrm{mol}$ ratios, respectively. Twenty thermal cycles $\left(4^{\circ}-50^{\circ} \mathrm{C}\right)$ were applied in order to ensure proper hydration and homogenization. The samples were extruded $\left(\right.$ at $60^{\circ} \mathrm{C}$ ) eight times through polycarbonate filters (Nucleopore, Whatman Inc.), first with $200 \mathrm{~nm}$ then $80 \mathrm{~nm}$ pore sizes using a LIPEX extruder (Northern Lipids Inc. Canada).

\section{Liquid chromatography-mass spectrometry (LC-MS)}

The molecular weight distribution of OMLA dissolved in water was analysed by liquid chromatography-mass spectrometry. LC-MS measurements were performed on a Sciex API2000 tandem mass spectrometer equipped with electrospray source. A Perkin Elmer Series 200 HPLC pump was used for solvent delivery system. Sample was introduced in flow injection mode into a water: acetonitrile flow (1:1). Flow rate of the eluent was $200 \mu 1 / \mathrm{min}$. Ions were detected in negative ion detection mode. Spray voltage was $-4800 \mathrm{~V}$. Source temperature was $300^{\circ} \mathrm{C}$. The LC-MS spectra show the occurrence of oligomer forms from dimer up to eleven units. The most intensive peaks correspond to 6 and 8 times of units. Monomer, however, was also present in low amount.

\section{Dynamic light scattering (DLS)}

The size distribution profile of SSVs was determined by an AvidNano W130i dynamic light scattering apparatus (AvidNano, UK). $80 \mu \mathrm{l}$ samples were used in a low-volume cuvette (UVette, Eppendorf Austria GmbH). The analysis of the measurement data was performed using the i-Size 3.0 software.

\section{Transmission electron microscopy combined with freeze fracture (FF-TEM)}

For freeze fracturing, an approximately $1 \mu \mathrm{l}$ droplet of the samples was pipetted onto golden sample holders and rapidly frozen in liquid freon, then transferred into liquid nitrogen [24]. Fracturing was performed at $-100^{\circ} \mathrm{C}$ in a Balzers freeze fracture device (Balzers BAF 400D, Balzers AG, Liechtenstein). The surface of samples were etched for $30 \mathrm{sec}$ at $-110^{\circ} \mathrm{C}$. The replicas, prepared by platinum-carbon shadowing, were cleaned with surfactant solution and washed by distilled water. From pure water the replicas were picked up on 200 mesh copper grids for examination in a Morgagni 268D (FEI, The Netherlands) transmission electron microscope.

\section{Small angle X-ray scattering measurements (SAXS)}

Small-angle X-ray scattering measurements were carried out at the ID02 beamline of the European Synchrotron Radiation Facility (ESRF, Grenoble, France) [25]. The energy of the incoming beam was $12 \mathrm{keV}$ and the two-dimensional scattering patterns were collected with a FReLoN 2000 CCD detector. The data were corrected for geometrical distortions, sample selfabsorption, instrumental background and normalized to primary beam intensity using the standard procedures on-site. Due to the lack of anisotropy in the samples on the investigated length scale, the scattering patterns were isotropic, therefore they were azimuthally averaged to yield one-dimensional scattering curves for each sample. The fitting of SAXS curves was carried out with a home-written Python-script similarly as described in [33]. 


\section{Fourier transform infrared spectroscopy (FTIR)}

ATR-FTIR (Attenuated Total Reflection Fourier Transform Infrared) spectra were collected on a Varian 2000 FTIR spectrometer (Varian Inc, USA) using a 'GoldenGate' single reflection diamond ATR accessory (Specac Ltd, UK). 256 scans were acquired at a resolution of $2 \mathrm{~cm}^{-1}$. Spectra were recorded at room temperature both as hydrated lipid suspension (immediately after pipetting a drop of the sample on the top of the ATR element) and as dry film spectra (after drying the sample on the ATR crystal). ATR correction was applied after each data collection. The spectral deconvolution of selected bands was performed using mixed Gaussian and Lorentzian functions. Band positions were estimated using the second derivative, band shapes were approximated by mixed Gaussian and Lorentzian function until the $\chi^{2}$ parameter became minimal. The GRAMS/32 software package was used for all spectral manipulations.

\section{Modelling the conformations and infrared spectra via computer simulations}

The Spartan program for molecular mechanics (MM) [26] was used for calculating frequencies to simulate IR spectra. Because of the large size of the model system, MM with MMFF force field was used instead of $a b$ initio methods (post-Hartree-Fock or density functional theory with sophisticated correlation calculations); in fact, the force field parameters are from ab initio calculations optimized for frequencies, so the MM calculation can be considered very accurate in our case. We were focusing on the $\mathrm{C}=\mathrm{O}$ functional groups of DPPC. These two groups yield characteristic bands in the spectra, and the different molecular environments have been found to have different effects on the peaks. The simulated system was a 2x2x1 DPPC chunk with either a linear pentameric OMLA (on the DPPC bilayer surface) or cholesterol (parallel insertion in the DPPC bilayer) or both in vacuum. The $2 \times 2 \times 1$ chunk model of the DPPC bilayer seems to be adequate in relation to IR spectra. The computationally expensive frequency calculation limits the size to be considered, and the other DPPC layer with opposite direction is far away from the functional groups considered.

\section{MTT cell viability test}

The viability of $\mathrm{H} 358$ cells was tested by MTT assay. H358 cells $\left(3 \times 10^{4} / \mathrm{ml}\right)$ were seeded onto 96-well plates in $100 \mu \mathrm{L}$ RPMI-1640 medium containing 10\% FBS. The cells were treated with three different systems (SSV, unilamellar DPPC vesicles loaded with OMLA, OMLA solution at indicated concentrations). After incubation for 72 hours, the culture medium was removed. $50 \mu \mathrm{L}$ sterile MTT dye was added to each well and incubated at $37^{\circ} \mathrm{C}$ for 1 hour. Finally, the MTT solution was removed and $200 \mu \mathrm{L}$ of developing medium ( $89 \%$ isopropanol, $10 \%$ triton $\mathrm{X} 100$, and $1 \% 0,1 \mathrm{~N} \mathrm{HCl}$ ) was added to dissolve the formazan crystals. The absorbance was measured at $570 \mathrm{~nm}$ using a microplate reader (Biotek II, Synergy, USA).

\section{Results and Discussion}

\section{Morphology and nanostructure}

Freeze-fracture TEM provides an excellent possibility to visualize the overall morphology of the vesicle systems. The two preparation procedures described in the Materials and methods resulted in two significantly different morphologies of concentrated multilamelar vesicles. When OMLA was added to the system in soluted state, the characteristic surface morphology of multilamellar vesicles were observed. On the other hand, when powders of OMLA and lipid crystals were hydrated together, anomalous heterodisperse and molten-like surface 
morphology of vesicles was observed, indicating the local hydrolytic effect of the low $\mathrm{pH}$ in the surroundings of just hydrated OMLA crystals. This explanation was confirmed by preparing DPPC vesicles in $\mathrm{HCl}$ solution, resulting in the same anomalous surface (details in Supplement 1). According to these findings, the solution of OMLA was further used for the preparation of unilamellar vesicles.

After extrusion uniform unilamellar vesicles were obtained with mean hydrodynamic diameters $\mathrm{D}_{\mathrm{m}}=102 \pm 15 \mathrm{~nm}$ and low polydispersity (P.d.=15.12\%). The DPPC, cholesterol and OMLA containing unilamellar vesicles were stable even after one month storage at $4{ }^{\circ} \mathrm{C}$.

The typical fractured surfaces of the nearly spherical vesicles are shown in Fig. 1 obtained after cooling - heating cycles. The fully hydrated lipid suspension consisting of dipalmitoylphosphatidylcholine, cholesterol and OMLA forms nearly spherical multilamellar vesicles as it can be observed in the electron micrographs in Fig. 1. Beside the large (over 2 $\mu \mathrm{m}$ diameter) multilamellar bodies (Fig. 1, left), closely packed small entities are also visible. The latter morphological observation indicates the ability of the system to form layer structures with small radii of curvature. The suspension was easily extruded and the white colour of the system turned into a nearly transparent state, confirming the absence of larger objects. The electron micrographs of the extruded system show spherical vesicles with a mean diameter below $100 \mathrm{~nm}$, corresponding to the pore size of the filter used (Fig. 1, right). The inset plot shows the fractured surface of the lipid bilayer of a vesicle broken through entirely, illustrating the existence of unilamellar formations.
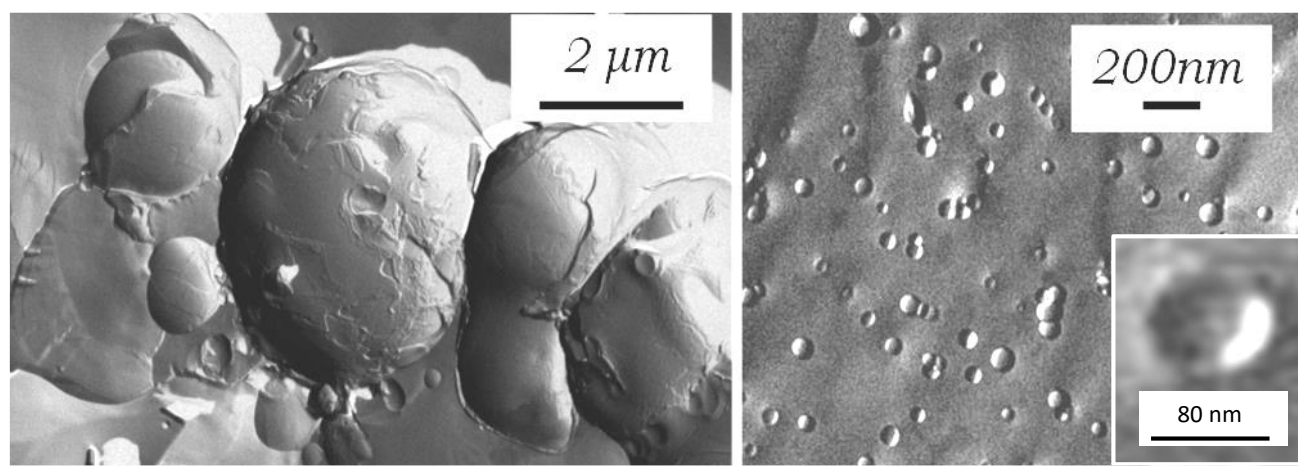

Fig. 1 Electron micrographs of freeze-fractured hydrated vesicles consisting of dipalmitoylphosphatidylcholine, cholesterol and OMLA; multilamellar vesicles before extrusion (left), unilamellar vesicles after extrusion (right)

Small angle X-ray scattering (SAXS) is a powerful method in describing both the overall size of the unilamellar vesicles and the fine structure of their bilayers [25, 27-29]. SAXS of the extruded vesicle system, carried out in a wide range of the scattering variable (q extending from $10^{-2}$ to $5 \mathrm{~nm}^{-1}$, where $\mathrm{q}=(4 \pi / \lambda) \cdot \sin \Theta$ is the scattering variable, $\Theta$ the half of the scattering angle and $\lambda$ the wavelength of the incident X-ray beam) yields complete structural and morphological information. The characteristic one-dimensional scattering curve of the hydrated lipid system having cholesterol and OMLA molecules (3:1 DPPC:cholesterol molar ratio, 100:5 DPPC:OMLA weight ratio) is shown in absolute units of scattering cross section as the function of the scattering variable, in Fig. 2. The bend in the scattering curve at the very small $q$-range (marked with $\mathrm{A}$ in the figure) characterizes the overall mean size of vesicles through the Guinier approximation [29]. Near q=0.1 1/nm a local maximum appears (B) which is a consequence of a relatively narrow size distribution. After a local minimum in the scattering curve a wide scattering peak (C) with two smaller ones (D and E) follows which carry information on the fine structure of the bilayer [30]. A thorough inspection reveals that the 
main peak $(\mathrm{C})$ is in fact composed of two or more double-layer components (marked with $\mathrm{F}$ and $\mathrm{G})$.

These features can be comprehensively interpreted by modelling the scattering curve using different mathematical models as shown in Fig. 3 [31]. The basis of the calculation is that the different parts of the bilayer (two head groups and one carbon chain region) can be described by different, characteristic electron-densities governed by the stoichiometry of their respective chemical constituents. Although step-like radial electron density functions would give adequate description for each region, Gaussian functions are more convenient to respect the thermal fluctuations of the constituents and the lack of sharp boundaries, which are not present in real systems. Taking into account the size distribution of vesicles with a log-normal distribution and modelling the electron density (ED) profile along the lipid bilayer normal with sum of Gaussian functions corresponding to the different regions, the theoretical SAXS curves were calculated using the characteristic parameters which we have determined for a similar system previously [32]. The simplest case of a monodisperse, spherical, unilamellar vesicle system already shows the main features of the measured scattering curve (left panel in Fig. 3). The calculated intensity starts with a decreasing, oscillating slope. The above mentioned Guinier region (marked with A) is also reproduced. The singularities on the scattering curve in the following range are the features of the scattering of spherical objects and are seen because of the monodisperse size distribution assumed in this case. The first local maximum (between the first two singularities), which is located at $\mathrm{q}=0.1 \mathrm{can}$ be recognized as the above mentioned shoulder in the experimental curve (B). The experimental curve does not contain further local maxima, indicating the polydispersity of the system. The broad peak $(\mathrm{C})$ is found between 0.4 and $2 \mathrm{~nm}^{-1}$ and the two further smaller ones (D and E) also appear as the envelope of a very strongly oscillating line. These are the results of the so-called bilayer form factor, i.e. the scattering contribution of a single bilayer.

In the experimental curve the broad peak $(\mathrm{C})$ can be separated into at least two subpeaks ( $\mathrm{F}$ and $\mathrm{G})$. These features can be added to our model by the assuming a periodic multi-layered structure, present only to a small extent. Supposing that the vesicles are oligolamellar with at most 5 bilayers, two relatively sharp diffraction peaks appear on the significantly extended form factor (middle panel of Fig. 3). Extending the model with a slightly heterodisperse size distribution and assuming that one tenth of the vesicles has two bilayers while the others are unilamellar yields more realistic scattering patterns, reconstructing all the features $(A, B, C, D$, E, F, G) of the experimental pattern (right panel of Fig. 3).

After these preliminary calculations the experimental curve was fitted in the least-squares sense, involving two identical, symmetrically placed Gaussian functions for the two head group regions, a third Gaussian for the carbon chain region and two further independent Gaussians for the guest molecules on the inner and outer side of the bilayer. The size distribution of the vesicles is assumed to follow the log-normal statistics. 


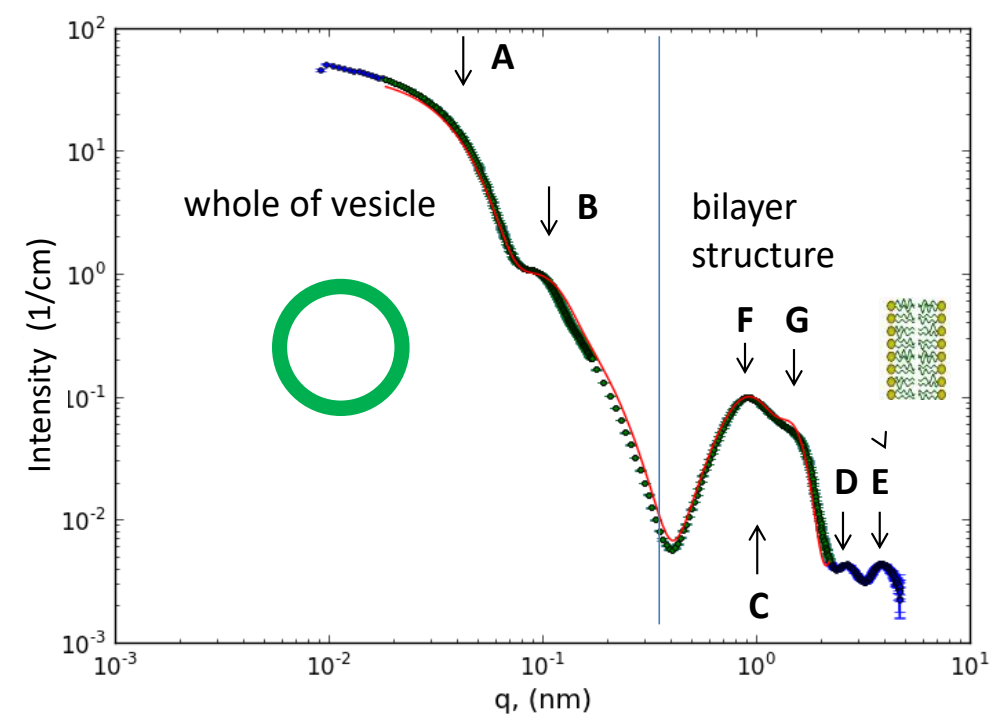

Fig. 2. Measured SAXS curve of the unilamellar vesicles system (symbols) and the leastsquares fitted model (continuous line). The characteristic parts of scattering curve are marked with letters (A: Guinier regime; B: first characteristic local maximum of monodisperse spherical vesicles; C: broad peak of the lipid bilayer form factor; D: second small peak of the lipid bilayer form factor; E: third small peak of the lipid bilayer form factor; F: first broad Bragg peak of two lipid bilayers, present in low amount; G: second broad Bragg peak of two lipid bilayers, present in low amount)
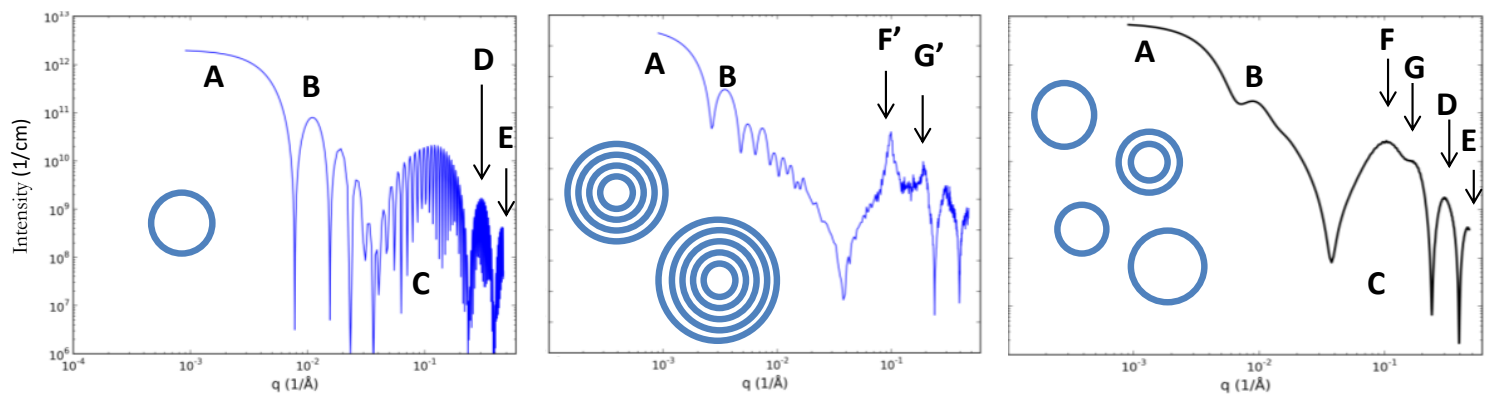

Fig. 3 Modelling the scattering curve of the unilamellar vesicle system. Monodisperse spherical unilamellar vesicles (left), slightly heterodisperse oligolamellar vesicles (mid), significantly heterodisperse mixture of uni- and bilamellar vesicles with a 9:1 ratio (right)

The best fitting model (continuous line in Fig. 2) corresponds to a nearly symmetric distribution of the electron density profile (shown in Fig. 4). The Gaussians representing the OMLA and cholesterol molecules are close to those representing the head groups, hinting that the respective molecules are localized close to the head region. The contribution of the guest molecules (OMLA and cholesterol) to the electron density seems to be different: the higher Gaussian at the outer side indicates a higher concentration of guest molecules than on the inner side of the double layer. This asymmetric distribution of the guest molecules shows similarities with the sterically stabilized unilamellar vesicles made with PEGylated lipids, and may originate from the difference of the positive and negative curvatures at the two sides [33]. When the ratio of OMLA increases (from 5 up to 10, related 100 lipid in w/w ratio) only a very small change can be observed in the shape of scattering profiles. The fit reveals that in the case of higher OMLA concentration the occurrence of two-bilayered (bilamellar) vesicles decreases from 12,5 down to $7,5 \%$ while the electron density of outer guest region is slightly increased. 
The fitted average outer radii of unilamellar vesicles are approx. $39 \mathrm{~nm}$ in both cases. Taking into consideration the bilamellar forms, the diameter of vesicles is nearly close to the size of pores of filters used during extrusion. The comparison of SAXS curves of the two systems and the summary of the fitting parameters can be found in Supplement 2.

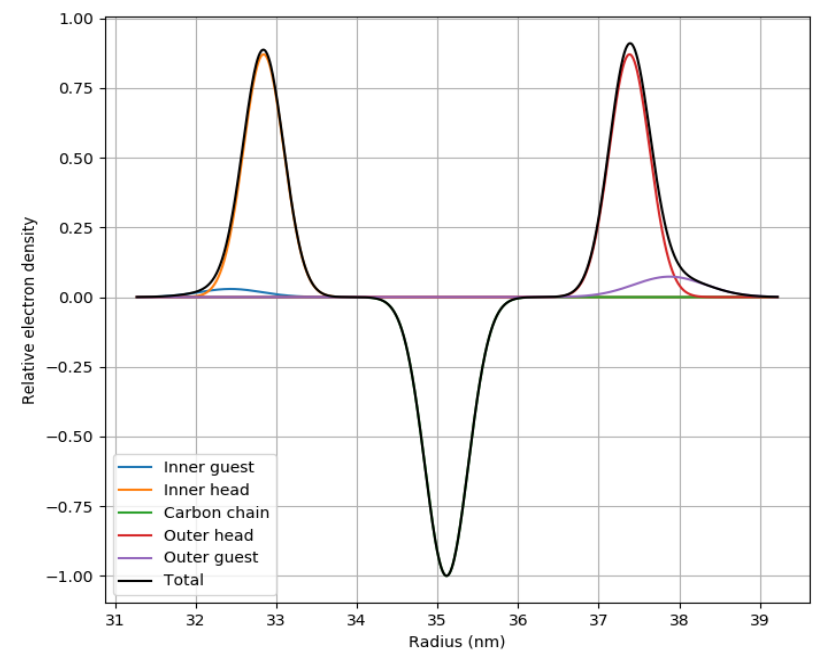

Fig. 4 Relative electron density profile of the lipid bilayer across the layer normal as determined from small-angle $\mathrm{X}$-ray scattering

\section{Interactions on atomic scale}

Infrared spectroscopy is a useful technique to examine the existence and the strength of localized interaction between molecules. Moreover, the IR spectrum provides information from all the three major regions of phospholipid molecules simultaneously. The polar headgroup region can be described by the phosphate $\left(1260-1000 \mathrm{~cm}^{-1}\right)$ and choline $\left(970 \mathrm{~cm}^{-1}\right)$ stretching vibrations; the polar-apolar interface is featured by the carbonyl $\mathrm{C}=\mathrm{O}$ stretching mode (1750$1720 \mathrm{~cm}^{-1}$ ) of the glycerol backbone; and finaly, the apolar acyl chain region might be characterized by the $\mathrm{CH}_{2}$ stretching and bending vibrations $\left(3000-2848 \mathrm{~cm}^{-1}\right.$ and $1468 \mathrm{~cm}^{-1}$, respectively).
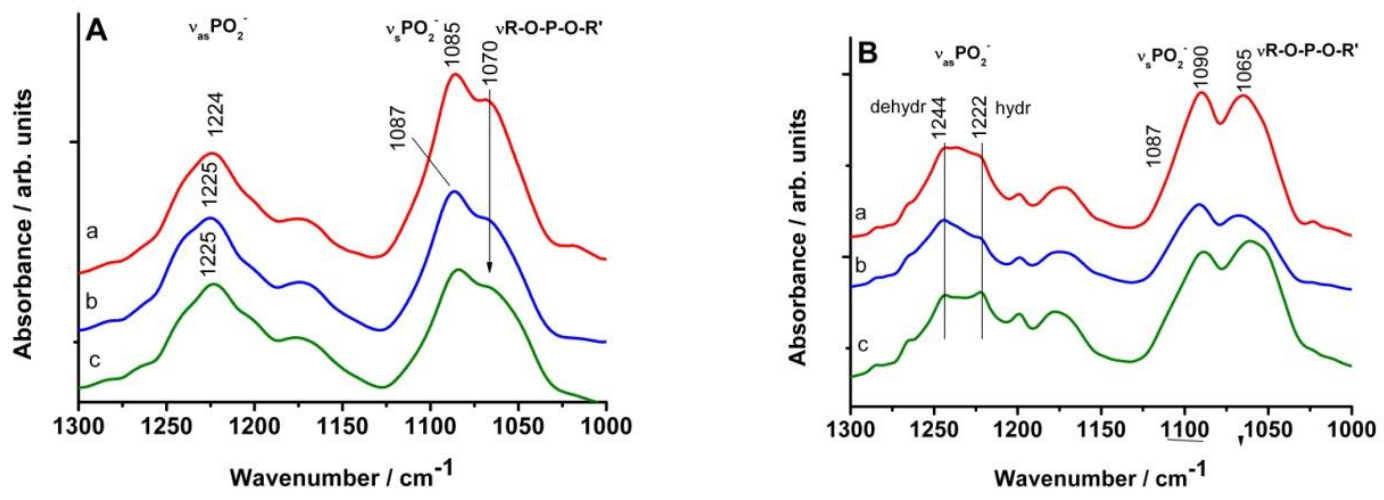

Fig. 5 Phosphate stretching spectral region of hydrated (A) and dry film (B) of DPPC-CHOLOMLA vesicles: a) DPPC:OMLA=100:0 ("blank"); b) DPPC:OMLA=100:5;

c) DPPC:OMLA=100:10 
Fig. 5 shows the regions of phosphate absorption bands containing antisymmetric $\left(v_{\mathrm{as}} \mathrm{PO}_{2}{ }^{-}\right.$at $\left.1250-1220 \mathrm{~cm}^{-1}\right)$ and symmetric $\left(v_{\mathrm{s}} \mathrm{PO}_{2}^{-}\right.$at $\left.1090-1080 \mathrm{~cm}^{-1}\right)$ stretching bands. The shoulder around $1070 \mathrm{~cm}^{-1}$ belongs to the R-O-P-O-R' stretching of phosphate diester and its change might be indicative for change in phosphate headgroup conformation towards lipid bilayer normal [34]. In the presence of OMLA the phosphate diester band ( $v \mathrm{R}-\mathrm{O}-\mathrm{P}-\mathrm{O}-\mathrm{R}$ ') intensity is suppressed. Simultaneous, both the antisymmetric $\left(v_{\mathrm{as}}\right)$ and symmetric $\left(v_{\mathrm{s}}\right) \mathrm{PO}_{2}{ }^{-}$stretching bands are shifted, implying a change in their molecular environment. Furthermore, the antisymmetric $\mathrm{PO}_{2}^{-}$stretching $\left(\mathrm{v}_{\mathrm{as}} \mathrm{PO}_{2}^{-}\right)$band is the most sensitive for the hydration state. Due to the H-bonded water molecules it has the maximum around $1224 \mathrm{~cm}^{-1}$ in fully hydrated state, while in dry state shifts toward higher wavenumbers $\left(\sim 1244 \mathrm{~cm}^{-1}\right)$. Using the dry film spectra method, i.e. waiting until the sample suspension will dry on the top of the diamond ATR crystal, the $\mathrm{H}$-bonded $\mathrm{PO}_{2}{ }^{-}$antisymmetric stretching band of phosphate moiety is still present (at 1222 $\mathrm{cm}^{-1}$ ) for vesicle containing OMLA at higher concentration. It seems plausible that a direct interaction exists between free -OH groups of OMLA and the $\mathrm{PO}_{2}^{-}$moiety of DPPC. Similar effect was observed also for OMLA-DPPC multilamellar vesicles [18]. The results of SAXS fit are in accordance with those obtained by IR insofar as both methods indicate the localization of guest molecules in vicinity of the lipid head groups.

The most notable effect of OMLA on DPPC structure may occur at the polar-apolar interface of the lipid bilayer. This region is represented by the stretching of ester carbonyl groups $(\vee C=O)$ around $1740 \mathrm{~cm}^{-1}$ (Fig. 6). In highly hydrated bilayers this band splits in two overlapping components: a high wavenumber band around $1742 \mathrm{~cm}^{-1}$ of the non-hydrogen bonded $\mathrm{C}=\mathrm{O}$ groups and a low wavenumber one around $1728 \mathrm{~cm}^{-1}$ due to the hydrogen bonding of the carbonyls. Formerly, these components were assigned solely to $s n-1$ and $s n-2$ ester bonds of the glycerol backbone [35-36]. Revised, these bands are assigned rather to differently hydrated subpopulations of both carbonyls [37]. Our previous experiences on multilamellar OMLA containing DPPC vesicles have shown no effect of OMLA on this spectral region in buffered samples. On the contrary, in the case of unilamellar vesicles the relative intensity (integral area) of the $\mathrm{H}$-bonded $\mathrm{vC}=\mathrm{O}$ band component is increased by the presence of OMLA. The results of curve fitting analysis are presented in Fig. 6. In the case of oligo(malic acid) containing unilamellar vesicles, the relative intensity of hydrogen bonded $\mathrm{C}=\mathrm{O}$ band (peak maximum around $1730 \mathrm{~cm}^{-1}$ ) becomes higher compared to the native DPPC-CHOL unilamellar vesicle sample.
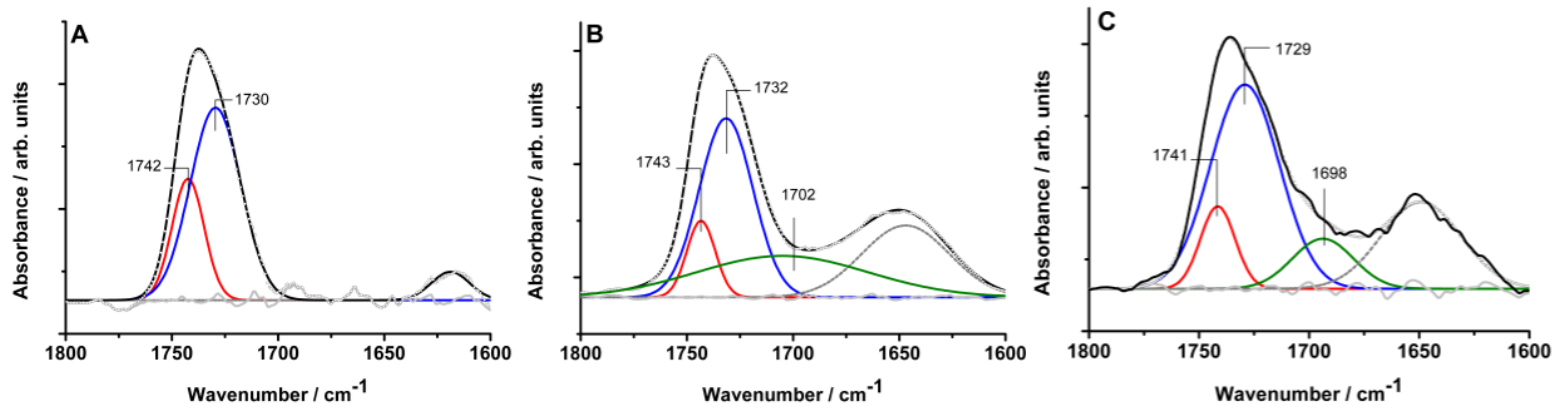

Fig. 6 ATR-FTIR spectra of the carbonyl stretching region $\left(1800-1600 \mathrm{~cm}^{-1}\right)$ of hydrated DPPC-CHOL-OMLA vesicles: A) DPPC ("blank"); B) DPPC:OMLA=100:5; C) DPPC:OMLA=100:10. The solid lines are the measured spectra; the red and blue lines are the fitted bands corresponding to intact and $\mathrm{H}$-bonded $\mathrm{C}=\mathrm{O}$ groups, respectively; the green lines are fitted bands belonging to the $\mathrm{C}=\mathrm{O}$ stretching of malic/oligomalic acid components; the 
dashed lines are fitted bands which might relate to fatty acids (impurities or hydrolysis results) and to residual water (inaccurate spectral subtraction).

Since cholesterol is embedded in the apolar lipid-region, it seems plausible that in case of ULVs the OMLA penetrate in the headgroup region of the lipid bilayer affecting the polar-apolar interface. Computer simulations using molecular mechanics (MM) approach strengthen this hypothesis.

The MM calculation was performed without water molecules. The calculated $\mathrm{C}=\mathrm{O}$ vibration frequencies are higher than the experimental ones. Those appear around $1850 \mathrm{~cm}^{-1}$ instead of the experimental values around $1730 \mathrm{~cm}^{-1}$. The $1730 / 1850=0.935$ is a plausible ratio, as well as the trend in the calculated frequencies, and are convenient for interpretation of molecular interactions in $\mathrm{C}=\mathrm{O}$ region (shown in Table 1). The selected $2 \times 2 \times 1$ chunk model of DPPC bilayer is respective for simulating the IR spectra.

Table 1. Calculated CO frequencies $\left(\mathrm{cm}^{-1}\right)$. The $2 \times 2 \times 1$ DPPC chunk is abbreviated as 4DPPC, the cholesterol with CHOL. For the $2 \times 2 \times 1$ DPPC chunk the average CO frequency was taken.

\begin{tabular}{|l|l|l|}
\hline & $\boldsymbol{s n}-\boldsymbol{1}$ & $\boldsymbol{s n}-\mathbf{2}$ \\
\hline DPPC & 1850 & 1844 \\
\hline 4 DPPC & 1848 & 1850 \\
\hline 4 DPPC+OMLA & 1848 & 1850 \\
\hline 4DPPC+CHOL & 1848 & 1849 \\
\hline 4DPPC+CHOL+OMLA & 1847 & $\mathbf{1 8 3 8}$ \\
\hline
\end{tabular}

In case of individual DPPC, the two not equivalent $\mathrm{C}=\mathrm{O}$ vibrations at 1844 and $1850 \mathrm{~cm}^{-1}$ correspond to the $s n-2$ (side chain) and $s n-1$ (main chain) ester carbonyl bonds of the glycerol backbone. In the self-assembled lipid molecules the difference of frequencies vanishes. The calculated frequencies for $\mathrm{C}=\mathrm{O}$ vibrations in the presence of oligo (malic acid) have the same values as for 4 DPPC, indicating that OMLA has no immediate interaction with the lipid $\mathrm{C}=\mathrm{O}$ groups. In the presence of cholesterol the frequency difference between $s n-2$ and $s n-1$ ester carbonyls practically disappears. This finding can be explained by the perturbed chain packing caused by the embedding of cholesterol guest-molecules. This loosed packing of DPPC molecules is shown in Fig. 7.

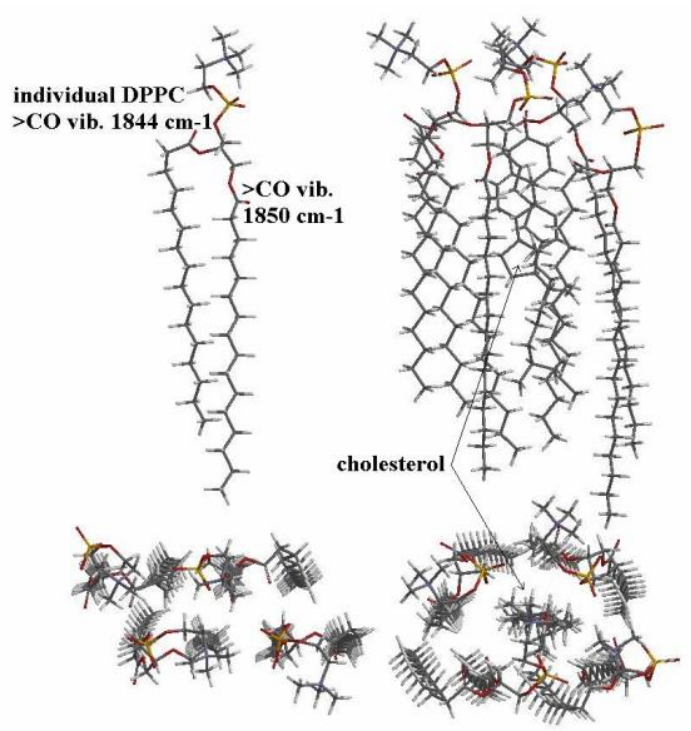


Fig. $7 \mathrm{MM}$ equilibrated packs of molecules are depicted. Individual DPPC (up left), the polar carbon side chain (sn-2) and apolar main chain (sn-1) each contains $\mathrm{CO}$ group (red), and the polar head group (outward from bilayer) contains $\mathrm{PO}_{2}{ }^{-}$group (yellow). View from above a $2 \times 2 \times 1$ chunk of DPPC layer: without (down left) and with embedded cholesterol (down right). The DPPC carbon chains and the cholesterol backbone are quasi-parallel (up and down right).

When cholesterol and OMLA molecules are present together in the lipid region, the frequency of $\mathrm{CO}$ band of $s n-2$ is drastically reduced, clearly indicating the consequence of location of OMLA molecules (Table 1). Beside the change of the characteristic frequency, the configuration of the calculated $2 \times 2 \times 1$ chunk of DPPC molecules with OMLA molecule is also altered as it is shown in Fig. 8. The OMLA is located deeper into the polar head region of DPPC when already cholesterol is embedded in the chain region of lipid layers.

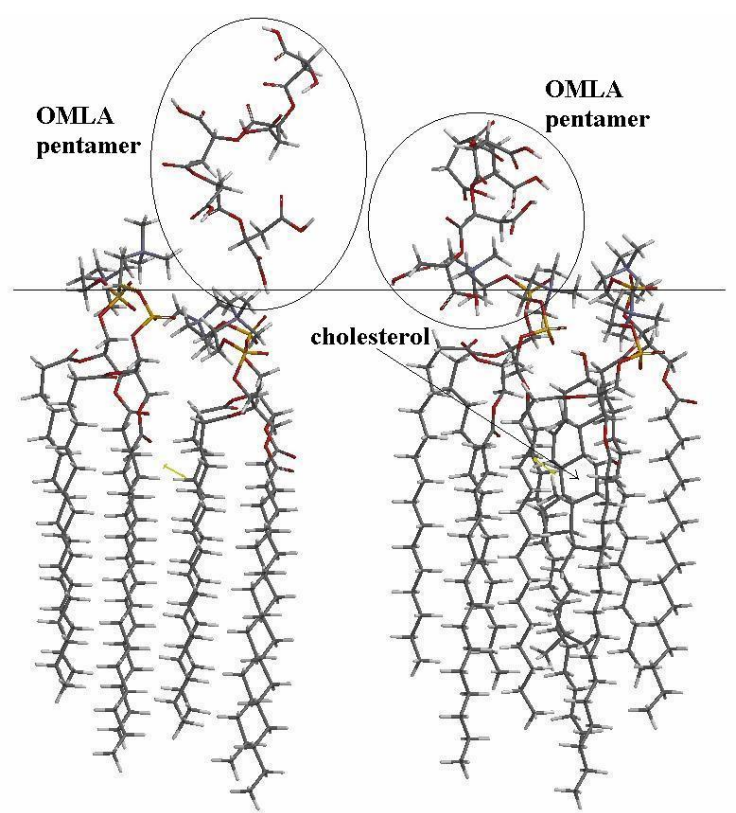

Fig. 8 Side view to an MM equilibrated $2 \times 2 \times 1$ chunk of DPPC with linear pentamer OMLA on its surface. When cholesterol is embedded in lipid chain region (right), the OMLA is embedded deeper in lipids, in comparison when cholesterol is not present (left). The horizontal line indicates the interface region of DPPC.

Moreover, a minor but peculiar effect of OMLA on the lipid order and packing of DPPCCHOL-OMLA vesicles was also noticed in the experimental IR spectra. The frequency of the peak position of the methylene symmetric stretching band $\left(\mathrm{v}_{\mathrm{s}} \mathrm{CH}_{2}\right)$ is a common parameter used to detect the conformational disorder and mobility of the hydrocarbon chain [38]. The presence of OMLA results in a slight shift of both antisymmetric $\left(v_{\mathrm{as}} \mathrm{CH}_{2}\right)$ and symmetric $\left(v_{\mathrm{s}} \mathrm{CH}_{2}\right)$ methylene stretching vibrations towards lower wavenumbers (Supplement 3), together with band narrowing, indicating a change in the acyl chain packing into a slightly more rigid form.

\section{Conclusion}

The complex structural study by a wide range of independent physico-chemical methods evidence the localization of oligo (malic acid) (OMLA) molecules to the polar region of lipid 
bilayers. Moreover, the OMLA molecules proved to be essential constituent of sterically stabilized unilamellar vesicles. The small-angle X-ray scattering reveals the asymmetrical displacement of these guest molecules. Detailed fine atomic structure of head regions revealed by ATR-IR spectroscopy and MM calculation implies that OMLA molecules connect to the polar head groups of lipids with well-defined van der Waals type interactions. During the OMLA-lipid connection a coherent interface (shell) region can be formed assuring a sterically stabilization of whole vesicles. In the evolvement of interfacial shell region, cholesterol also plays a significant role. In the interaction between lipid polar headgroup and OMLA cholesterol acts as a spacer, mellows the chain region of lipid matrix, thereby it invigorates the entering of OMLA deep into the polar region of lipids. Both guest molecules (OMLA and cholesterol) increase the rigidity of the membranes as it was concluded from the shift of antisymmetric and symmetric methylene stretching vibrations of lipid acyl chains. Consequently, the OMLA and cholesterol provide the increased stabilization of vesicles generally.

Furthermore, in the OMLA-stabilized SSVs the presence of oligo(malic acid) molecules brings forth a low $\mathrm{pH}$ regime that is even tolerable against lipid hydrolysis. In certain local milieu, where lower $\mathrm{pH}$ value may exist, an enhanced hydrolysis and through this a $\mathrm{pH}$ sensitive character can be supposed giving the possibility for a $\mathrm{pH}$ sensitive targeting.

Barbosa et al. investigated the cytotoxicity of poly(malic acid)-based nanoparticles and the toxic effect of the degradation products [39]. They found a relation between the toxic effect and the rate of poly (malic acid) degradation. The latter produce low molecular weighted fractions, which can really induce drastic local effects as it was observed in case of model lipid system, too and shown in Supplement 1. In our case, the OMLA molecules are present in low molar ratio, and connected to polar head regions of lipid bilayers. It seems plausible that their release is slow vanishing any toxic effect. A preliminary in vitro toxicity assay on cancer cell line (H358) [40] showed no significant cytotoxicity both of OMLA and of the novel OMLAstabilized vesicle up to $250 \mu \mathrm{g} / \mathrm{mL}$ concentration (Supplement 4). Nevertheless, further study investigating the release and fate of OMLA in biological-relevant medium is envisaged to prove the applicability of the OMLA-stabilized SSVs as potential new nanocarriers.

\section{Acknowledgements}

The work was supported by the János Bolyai Research Scholarship of the Hungarian Academy of Sciences (J.M. and Z.V.) and the Hungarian Scientific Research Found (OTKA, Hungary) and the National Innovation Office (NIH, Hungary) under grant agreement CNK-81056. Teréz Kiss is gratefully acknowledged for performing the FF-TEM pictures. We are grateful to the European Synchrotron Radiation Facility for provision of beam time at ID02 (proposal no. SC3269 , Z.V.).

\section{References:}

[1] D. Papahadjopoulos, T.M. Allen, A. Gabizon, E. Mayhew, K. Matthay, S.K. Huang, K-D. Lee, M.C. Woodle, D.D. Lasic, C. Redemann, J.F. Martin, Sterically stabilized liposomes: improvements in pharmacokinetics and antitumor therapeutic efficacy, Proc. Natl Acad. Sci. USA, 88, (1991) 11460-11464.

[2] M. C. Woodle, Sterically stabilized liposome therapeutics, Adv. Drug Deliv. Rev. 16, (1995) 249-265. 
[3] K. Nag Okhil and A. Vibhudutta, Surface Engineering of Liposomes for Stealth Behavior, Pharmaceutics 5 (2013) 542-569; doi:10.3390/pharmaceutics5040542]

[4] A.D.Bangham, R.W. Horne, Negative Staining of Phospholipids and Their Structural Modification by Surface-Active Agents As Observed in the Electron Microscope, Journal of Molecular Biology. 8 (5) (1964) 660-668.

[5] D.D. Lasic, Doxorubicin in sterically stabilized liposomes, Nature 380 (1996) 561-562.

[6] Y. Barenholz, Liposome application: problems and prospects. Curr. Opin. Colloid Interface Sci. 6 (2001) 66-77.

[7] H-I. Chang, M-K. Yeh: Clinical development of liposoma-based drugs: formulation, characterization, and therapeutic efficacy, Int. J. of Nanomedicine, Review, 7 (2012) 49-60.

[8] J. Szebeni, Complement activation-related pseudoallergy: a new class of drug-induced acute immune toxicity, Toxicology, 216(2-3) (2005) 106-121.

[9] M. Rinaudo, F. Quemeneur, B. Pépin-Donat , Stabilization of Liposomes by Polyelectrolytes: Mechanism of Interaction and Role of Experimental Conditions, Macromol. Symp. 278 (2009) 67-79. [10] M. Rinaudo, F. Quemeneur, B. Pépin-Donat, Experimental characterization of liposomes stabilized by polyecetrolytes and mechanism of interaction involved, International Journal of Polymer Anal. Charact., 17 (2012) 1-10.

[11] D. Peer, A. Florentin, R. Margalit, Hyaluronan is a key component in cryoprotection and formulation of targeted unilamellar liposomes, Biochim. et Biophys. Acta - Biomembranes 1612 (2003) 76-82.)

[12] D. Peer, R. Margalit, Tumor-targeted hyaluronan nanoliposomes increase the antitumor activity of liposomal Doxorubicin in syngeneic and human xenograft mouse tumor models, Neoplasia, 6 (2004) 343-353.

[13] Na-Kyung Han, Dae Hwan Shin, Jung Seok Kim, Kwon Yeon Weon, Chang-Young Jang, Jin-Seok Kim, Hyaluronan-conjugated liposomes encapsulating gemcitabine for breast cancer stem cells, Int J Nanomedicine, 11 (2016) 1413-1425.

[14] J.Y. Ljubimova, M.Fujita, A.V. Ljubimov, V.P. Torchilin, K.L. Black, E. Holler: Poly(malic acid) nanoconjugates containing various antibodies and oligonucleotides for multitargeting drug delivery, Nanomedicine (London) 3 (2008) 247-265.

[15] Z. W. Huang, V. Laurent, G. Chetouani, J.Y. Ljubimova, E. Holler, T. Benvegnu, P. Loyer, S. Cammas-Marion, New functional degradable and bio-compatible nanoparticles based on poly(malic acid) derivatives for site-specific anti-cancer drug delivery, Int. J. Pharm. 423 (2012) 84-92.

[16] M. Vert, R. W. Lenz, Preparation and properties of poly-beta-malic acid: A functional polyester of potential biomedical importance, ACS Polym. Preprints 20 (1979) 608-611.

[17] M. Vert, Chemical routes to poly $(\beta$-malic acid) and potential applications of this watersoluble bioresorbable poly( $\beta$-hydroxy alkanoate), Polym. Degrad. Stab., 59 (1998) 169-175. 
[18] Sz. Berényi, J. Mihály, S. Kristyán, L. Naszályi Nagy, J. Telegdi, A. Bóta: Thermotropic and structural effects of poly(malic acid) on fully hydrated multilamellar DPPC-water systems, Biochemica et Biophysica Acta- Biomembranes, 1828 (2013) 661-669.

[19] A.S. Kaviratna, R. Banerjee: The effect of acids on dipalmitoyl phosphatidylcholine (DPPC) monolayers and liposomes. Colloid Surface A 354 (2009) 155-162.

[20] S. General, A.F. Thünemann: pH-sensitive nanoparticles of poly(amino acid) dodecanoate complexes. Int. J. Pharm. 230 (2011) 11-24.

[21] T. Kajiyama, T. Taguchi, H. Kobayashi, K. Kataoka, J. Tanaka, Synthesis of high molecular weight poly $(\alpha, \beta$-malic acid) for biomedical use by direct polycondensation, Polym. Degrad. Stab. 81 (2003) 525-530.

[22] T. Kajiyama, H. Kobayashi, T. Taguchi, K. Kataoka, J. Tanaka, Improved Synthesis with High Yield and Increased Molecular Weight of $\operatorname{Poly}(\alpha, \beta$-malic acid $)$ by Direct Polycondensation, Biomacromol. 5 (2004) 169-174.

[23] V.P. Torchilin, V. Weissig ed. Liposomes: A practical approach; Oxford University Press 2003.

[24] H.W.Meyer, W. Richter, Freeze-fracture Studies on Lipids and Membranes, Micron 32(6) (2001) 615-644.

[25] T. Narayanan, O. Diat and P. Boesecke, Nucl. Instrum.Methods Phys. Res. Sect. A, 467 (2001) 1005-1009.

[26] B.J. Deppmeier, A.J. Driessen, T.S. Hehre, W.J. Hehre, J.A. Johnson, P.E. Klunzinger, J.M. Leonard, I.N. Pham, W.J. Pietro, Jianguo Yu: Wavefunction SPARTAN '06, build 129 (Jun 8 2007)

[27] O. Glatter, and O. Kratky, "Small angle x-ray scattering", New York: Academic Press, 1982.

[28] A. Feigin, and D. I. Svergun, "Structure Analysis by Small-Angle X-Ray and Neutron Scattering”, New York: Plenum Press, 1987.

[29] A. Guinier, and G. Fournet, "Small-angle scattering of X-rays", New York: John Wiley \& Sons Inc., 1955.

[30] R. Zhang, R. M. Suter, J.F. Nagle, Theory of the structure factor of lipid bilayers, Phys. Rev. E 50 (1994) 5047-5060.

[31] G. Pabst, R., Koschuch, B. Pozo-Navas, M. Rappolt, K. Lohner, P. Laggner, Structural analysis of weakly ordered membrane stacks. J. Appl. Cryst. 36 (2003)1378-1388.

[32] Z. Varga, Sz. Berényi, B. Szokol, L. Örfi, Gy. Kéri, I. Peták, A. Hoell and A. Bóta: A Closer Look at the Structure of Sterically Stabilized Liposomes: A Small -Angle X-Ray Scattering Study, Journal of Physical Chemistry B, 114 (2010)6850-6854. 
[33] Z. Varga, A. Wacha, U. Vainio, J. Gummel and A. Bóta: Characterization of the PEG layer of sterically stabilized liposomes: a SAXS study, Chemistry and Physics of Lipids, 165, 4 (2012) 387-392.

[34] J.L.R Arrondo, F.N. Goni, J. M. Maccarulla, Infrared spectroscopy of phosphatidylcholines in aqueous suspension: a study of the phosphate group vibrations, Biochimica et Biophysica Acta (BBA) - Lipids and Lipid Metabolism, 794, 1 (1984), 165-168.

[35] I.W. Levin, E. Mushayakarar, R. Bittman, Vibrational assignment of the $s n-1$ and $s n-2$ chain carbonyl stretching modes of membrane phospholipids, J Raman Spectr, 13, 3 (1982) 231-234.

[36] H. Hauser, I. Pascher, R.H. Pearson, S. Sundell., Preferred conformation and molecular packing of phosphatidylethanolamine and phosphatidylcholine, Biochim Biophys Acta. 650 (1981) 21-51.

[37] R.N.A.H. Lewis, R.N. McElhaney, W. Pohle, H.H. Mantsch, Components of the carbonyl stretching band in the infrared spectra of hydrated 1,2-diacylglycerol bilayers: A reevaluation, Biophys J 67 (1994) 2367-2375.

[38] Z.D. Schultz, I.W.Levin, Vibrational Spectroscopy of Biomolecules, Annu. Rev. Anal. Chem. 4 (2011) 343-66

[39] M.E. Martinez Barbosa, S. Cammas, M. Appel, G. Ponchel: Investigation of the degradation mechanisms of poly(malic acid) esters in vitro and their related cytotoxicities on J774 macrophages. Biomacromolecules 5 (2004) 137-143

[40] S. Maassen, E. Fattal, R. Muller, P. Couvreur: Cell cultures for the assessment of toxicity and uptake of polymeric drug carriers. S.T.P. Pharma Sci. 3 (1993) 11-22. 